\title{
Inhaled corticosteroids should be effective for COVID-19 treatment
}

\author{
Daisuke Miyazawa ${ }^{1}$ and Gen Kaneko ${ }^{1}$ \\ ${ }^{1}$ Affiliation not available
}

July 8, 2020

Several recent studies have reported that systemic corticosteroids were effective against COVID-19. Horby et al. reported that systemic dexamethasone was effective in patients with severe COVID-19 who were receiving oxygen or ventilator support. ${ }^{\mathbf{1}}$ Patients with severe COVID-19 present with acute respiratory distress syndrome (ARDS), also known as diffuse alveolar damage, presumably caused by an excessive immune response in the alveoli. However, although systemic corticosteroids may be effective against excessive alveolar inflammation, there are concerns about systemic side effects such as opportunistic infections, delayed viral elimination, and osteoporosis, similar to those observed following the use of corticosteroids for the treatment of patients with SARS-CoV infection in 2003.

Similar but milder alveolar inflammation should also exist in early stage patients. Systemic corticosteroids for patients with early stage COVID-19 may improve prognosis by suppressing excessive inflammation in the alveoli, but adverse effects such as delayed systemic elimination of the virus due to systemic immunosuppression may outweigh this advantage. This could be the reason why the study by Horby et al. found no benefit in less severe patients who were not receiving oxygen. ${ }^{1}$ SARS-CoV-2 infection is suggested to elicit inflammatory cytokine secretion from alveolar epithelial type 2 cells. ${ }^{2}$ If it is assumed that a steroid's efficacy for COVID-19 is in some part due to the suppression of excessive inflammation in the alveoli, then delivering corticosteroids directly to the alveoli by inhalation should also be effective and would have fewer systemic side effects. This advantage could benefit not only early stage but also severe patients. Additionally, ICS (inhaled corticosteroids) deposited in the alveoli enters the systemic circulation via pulmonary vasculature, leading to potential anti-inflammatory effects on pulmonary vasculature, where inflammation and subsequent microthrombosis are considered likely to occur in COVID-19.

The use of systemic corticosteroids for ARDS is controversial, possibly because the main cause of ARDS is bacterial infection and immunosuppression can be harmful. Nebulized budesonide improved oxygenation and significantly reduced inflammatory markers (TNF- $\alpha$, IL- $1 \beta$, and IL-6) in patients with ARDS. ${ }^{3}$ These three inflammatory markers have also been reported to be elevated in patients with COVID-19 and have been linked to the severity of the disease.

The results of a study that investigated the association between regular ICS use and COVID-19 mortality did not support the hypothesis that ICS protect against COVID-19-related deaths. ${ }^{4}$ However, the study also indicated that the observed harmful associations could be readily explained due to underlying health differences between people who used ICS regularly, for asthma and COPD, and those that did not, rather than as a causally harmful effect of ICS.

ICS can reach different sites in the lungs depending on particle size. ${ }^{5}$ Since the alveoli are considered to be the main site for lung inflammation in COVID-19, steroids with smaller particle sizes that reach the alveoli should be more promising. Among ICS, pressurized metered-dose inhalers (pMDI) of beclomethasone and ciclesonide have the smallest particle sizes $(<2 \mu \mathrm{m})$ and hence are considered to reach the alveoli more easily. ${ }^{\mathbf{5}}$ The particles in the nebulizer are also small enough to reach the alveoli, but there is a concern that the particles that return on exhalation may contain the virus and could infect medical personnel. 
Apart from their anti-inflammatory effects, some ICS have been found to have antiviral effects. Ciclesonide and mometasone, which are both marketed as ICS, suppressed the replication of SARS-CoV-2 and MERSCoVin vitro, whereas dexamethasone, cortisone, prednisolone, and fluticasone did not. ${ }^{6}$ There is a case report of three COVID-19 patients treated with inhaled ciclesonide. ${ }^{7}$ Although it might be more difficult for dry powder inhalers (DPI) to reach the alveoli, owing to their larger particle size, than for pMDIs, ${ }^{\mathbf{5}}$ mometasone may be worth considering, as it has antiviral properties and a smaller particle size than budesonide. ${ }^{\mathbf{5}} \mathrm{A}$ mutant MERS-CoV that developed resistance to ciclesonide did not show resistance to mometasone. ${ }^{6}$

As of July 5, 2020, several clinical trials worldwide utilizing ICS for COVID-19 have been registered on ClinicalTrials.gov: 4 trials ( 1 recruiting, 3 not yet) for ciclesonide and 4 trials (3 recruiting, 1 not yet) for budesonide (1 including formoterol).

Since ICS, theoretically, have a high potential to reduce harmful alveolar inflammation in patients with COVID-19, have few systemic side effects, and can be readily used in outpatients, we conclude that ICS should be looked at as a promising therapeutic candidate and should be prioritized for clinical trials in both mildly symptomatic outpatients and severely ill inpatients. The antiviral effect of ICS, as well as their particle size, which is related to the ability to reach the alveoli, should also be noted. We propose that not only clinical trials that confirm the clinical effect of beclomethasone, which has a similar particle size to ciclesonide, but studies that confirm the antiviral effect of it, should be conducted. This is partly because if either or both drugs are found to be effective, it may be possible to determine whether these effects are due to the antiviral or anti-inflammatory effect. For the same reason, we propose that clinical trials of mometasone, which has antiviral properties and a smaller but similar particle size to budesonide, should also be conducted.

\section{Acknowledgments}

Author contributions: Both DM and GK contributed to the conception and design of the work; the acquisition, analysis, and interpretation of data; and drafting and revision of the manuscript. DM and GK approved the current version of the manuscript and agree to be accountable for all aspects of the work.

Disclosure: The authors declare no conflicts of interest.

\section{References}

1. Horby P, Lim WS, Emberson J, et al. Effect of dexamethasone in hospitalized patients with COVID-19: preliminary report. medRxiv 2020.06.22.20137273; doi:https://doi.org/10.1101/2020.06.22.20137273

2. Huang J, Hume AJ, Abo KM, et al. SARS-CoV-2 Infection of pluripotent stem cell-derived human lung alveolar type 2 cells elicits a rapid epithelial-intrinsic inflammatory response. bioRxiv 2020.06.30.175695; doi: https://doi.org/10.1101/2020.06.30.175695

3. Mohamed HS, Meguid MM. Effect of nebulized budesonide on respiratory mechanics and oxygenation in acute lung injury/acute respiratory distress syndrome: Randomized controlled study. Saudi J Anaesth. 2017;11(1):9-14. doi:10.4103/1658-354X.197369

4. Schultze A, Walker AJ, MacKenna B, et al. Inhaled corticosteroid use and risk COVID-19 related death among 966,461 patients with COPD or asthma: an OpenSAFELY analysis. medRxiv 2020.06.19.20135491; doi:https://doi.org/10.1101/2020.06.19.20135491

5. Nave R, Mueller H. From inhaler to lung: clinical implications of the formulations of ciclesonide and other inhaled corticosteroids. Int J Gen Med. 2013;6:99-107. doi:10.2147/IJGM.S39134

6. Matsuyama S, Kawase M, Nao N, et al. The inhaled corticosteroid ciclesonide blocks coronavirus RNA replication by targeting viral NSP15. bioRxiv 2020.03.11.987016; doi:https://doi.org/10.1101/2020.03.11.987016

7. Iwabuchi K, Yoshie K, Kurakami Y, et al. Therapeutic potential of ciclesonide inhalation for COVID-19 pneumonia: Report of three cases. J Infect Chemother. 2020;26(6):625-632. doi:10.1016/j.jiac.2020.04.007 